overweight and obesity in early adulthood. The specific aim of this study was to assess how parents' perception of neighborhood safety is associated with PA in children aged 10-12 years. Methods: Based on a cross-sectional design, 201 parent-child dyads were enrolled from a random sample of Kaiser Permanente members in two distinct geographic areas in Oakland, California, designed to represent relatively high and low SES based on zip code and census tract information. Parents and children were interviewed by telephone and asked their perception of the neighborhood environment (a modification of the Neighborhood Environment Walkability Survey (NEWS)) including factors related to traffic and crime safety, social capital, and aesthetics, and about frequencies of specific crimes. For each domain, a summary score was created, which was the mean of the score of all items in the domain. Parents reported the number of days/week their child participated in vigorous and moderate PA. Ordered logistic regression was used to model the association between the NEWS/crime frequency variables and parents' report of children's vigorous and moderate physical activity. Analyses were stratified by high-income versus low-income zip codes and adjusted for child's gender, parental SES variables and race/ethnicity. For each model, we obtained the odds ratio, 95\% confidence interval, $P$-value, and q-value to adjust for repeated testing in each domain. Results: In the adjusted model, items from the NEWS that were significantly associated with parents' report of children's vigorous PA included safety of parks at night and the presence of crosswalks and pedestrian signals. The crime frequency summary score and frequency of some specific crimes (i.e., murder, being beaten up, teens carrying guns) were also associated with children's vigorous PA. Conclusions: The results of this study indicate the importance of considering parents' concern for children's safety in the design of programs, interventions, health messages and counseling that aim to promote children's physical activity.

\section{Abstract PS1-31 \\ The Association Between Body Mass Index and High Sensitivity C-Reactive Protein by Gender}

H. Lester Kirchner, PhD, Geisinger Center for Health Research; Nirav R. Shah, MD, MPH, Geisinger Center for Health Research; J.B. Jones, MBA, PhD, Geisinger Center for Health Research; G. Craig Wood, MS, Geisinger Center for Health Research; Zahra Daar, MS, Geisinger Center for Health Research; Walter F. Stewart, PhD, MPH, Geisinger Center for Health Research

Background/Aims: Prior studies relating inflammatory biomarkers to obesity demonstrated a correlation between elevated body mass index (BMI) and elevated C-reactive protein (CRP). To date, however, little research has examined the association of the newer, high-sensitivity CRP assay (hs-CRP) to BMI of both men and women. Methods: In this cross-sectional study, the association between BMI and hs-CRP by gender was estimated in 376 subjects recruited from a network of community-based, primary care clinics in eastern Pennsylvania. The data were analyzed with Spearman's correlation and multivariate linear regression, accounting for age, gender, high density lipoprotein, and hypertension. The natural logarithmic transformation was used due to the presence of right-skewness of the distribution of hs-CRP. Results: All subjects were Caucasian, $52 \%$ were male with a mean age of 62 , BMI of 30.6. The sample consisted of $21 \%$ with diabetes mellitus and $72 \%$ with hypertension. There was a significant positive association between BMI and hs-CRP $(r=0.28)$ for the entire sample. This association was slightly weaker in males $(\mathrm{r}=0.24)$ compared to females $(\mathrm{r}=0.31)$, and did not significantly vary $(P=0.9425)$. The adjusted associations demonstrated limited confounding (males: $\mathrm{r}=0.23$; females: $\mathrm{r}=0.28$ ), suggesting that $\mathrm{BMI}$ may be an independent factor for elevated hs-CRP. Conclusions: BMI and hs-CRP correlate positively, slightly more for women than men. In patients with elevated BMI, elevated hs-CRP has potential for use as a cardiac risk stratification tool. Elevated levels of hs-CRP in the obese individual could direct initiation of statin use to reduce the chronic inflammatory state or assist in cardiac risk stratification. In a time of limited patient resources and tighter budgets, these data give promise for the judicious use of biomarkers based on BMI for cardiac risk stratification.
Abstract PS1-32

Attorney General Consumer and Prescriber Grant Program. Changing Prescriber Behavior: The Meyers Primary Care Experience

Melissa A. Fischer, MD, MEd, Meyers Primary Care Institute and Fallon Clinic Foundation and Fallon Community Health Plan and University of Massachusetts Medical School; Mary Ellen Keough, MPH, Meyers Primary Care Institute and Fallon Clinic Foundation and Fallon Community Health Plan and University of Massachusetts Medical School; Joann L. Baril, BS, Meyers Primary Care Institute and Fallon Clinic Foundation and Fallon Community Health Plan and University of Massachusetts Medical School; Thomas McLaughlin, ScD, University of Massachusetts Medical School; Becky Briesacher, PhD, University of Massachusetts Medical School; Michael Tutty, MHA, University of Massachusetts Medical School; Jennifer L. Donovan, PharmD, Massachusetts College of Pharmacy and Health Sciences; Mark Quirk, EdD, University of Massachusetts Medical School; Terry S. Field, DSc, Meyers Primary Care Institute and Fallon Clinic Foundation and Fallon Community Health Plan and University of Massachusetts Medical School; Kathleen M. Mazor, EdD, Meyers Primary Care Institute and Fallon Clinic Foundation and Fallon Community Health Plan and University of Massachusetts Medical School; Jerry H. Gurwitz, MD, Meyers Primary Care Institute and Fallon Clinic Foundation and Fallon Community Health Plan and University of Massachusetts Medical School

Background: The Meyers Primary Care Institute (MPCI) is one of 28 Attorney General Consumer and Prescriber Grant Program grantees. Four HMORN CERTs sites, including MPCI, received grants. The Institute is developing curricular interventions to educate prescribers regarding the influence of pharmaceutical marketing on prescribing behavior. Our objectives are to encourage prescribers to incorporate understanding of this influence into their practice and utilize best evidence to improve patient care. Methods: In 2006-2007 the MPCI convened a multidisciplinary group of advisors with skills in research, patient care and education. We convened focus groups of prescribers (NP, PA, PharmD and MD) to learn about their experiences with pharmaceutical representatives. Integrating this evidence with detailed data from the peer reviewed literature and recommendations regarding best-practices in electronic learning, we are developing 6 educational modules: (1) Pharmaceutical Development and Regulation; (2) Evidence-Based Medicine; (3) Pharmaceutical Marketing; (4) Organizational Influence; (5) Communication with Pharmaceutical Representatives; and (6) Communication with Patients. These modules will be freely accessible to all prescribers via web-based delivery systems, will include downloadable PowerPoint presentations and content notes for faculty who wish to integrate them into their own curricula, and PDA downloads for clinicians. Results: We will showcase elements from the modules, discuss issues of development and implementation, and suggest ways in which HMORN partners may be able to utilize these and other grantee resources to improve the practice of their clinical partners.

Abstract PS1-36

Secular Trends in Diagnosis and Antibiotic Treatment of Childhood Infections

Virginia L. Hinrichsen, MS, MPH, Department of Ambulatory Care and Prevention, Harvard Medical School \& Harvard Pilgrim Health Care; Jonathan A. Finkelstein, MD, MPH, Department of Ambulatory Care and Prevention, Harvard Medical School \& Harvard Pilgrim Health Care; Sheryl L. Rifas-Shiman, MPH, Department of Ambulatory Care and Prevention, Harvard Medical School \& Harvard Pilgrim Health Care; Ken Kleinman, ScD, Department of Ambulatory Care and Prevention, Harvard Medical School \& Harvard Pilgrim Health Care; Susan S. Huang, MD, $\mathrm{MPH}$, University of California Irvine School of Medicine

Background: Antibiotic prescribing rates for young children have declined dramatically in recent years. We aimed to determine what drove this decline in 8 communities throughout Massachusetts. Methods: We obtained automated claims data from four large health insurers on antibiotic dispensings and visit diagnoses in children 3 to $<24$ months of age and 24 to $<48$ months of age from Sept 1998-August 2003, and created diagnosis categories for common infections. We determined diagnosis rates (\# visits for each diagnosis/total \# of person-years [p-y]), antibiotic treatment rates (\# visits for each diagnosis associated with an antibiotic/ total \# of p-y) and the percent 